\title{
Systems biology approach reveals that overflow metabolism of acetate in Escherichia coli is triggered by carbon catabolite repression of acetyl-CoA synthetase
}

\author{
Kaspar Valgepea ${ }^{1,2}$, Kaarel Adamberg ${ }^{2,3}$, Ranno Nahku ${ }^{1,2}$, Petri-Jaan Lahtvee ${ }^{1,2}$, Liisa Arike ${ }^{2,3}$, Raivo Vilu ${ }^{1,2^{*}}$
}

\begin{abstract}
Background: The biotechnology industry has extensively exploited Escherichia coli for producing recombinant proteins, biofuels etc. However, high growth rate aerobic E. coli cultivations are accompanied by acetate excretion i.e. overflow metabolism which is harmful as it inhibits growth, diverts valuable carbon from biomass formation and is detrimental for target product synthesis. Although overflow metabolism has been studied for decades, its regulation mechanisms still remain unclear.
\end{abstract}

Results: In the current work, growth rate dependent acetate overflow metabolism of E. coli was continuously monitored using advanced continuous cultivation methods (A-stat and D-stat). The first step in acetate overflow switch (at $\mu=0.27 \pm 0.02 \mathrm{~h}^{-1}$ ) is the repression of acetyl-CoA synthethase (Acs) activity triggered by carbon catabolite repression resulting in decreased assimilation of acetate produced by phosphotransacetylase (Pta), and disruption of the PTA-ACS node. This was indicated by acetate synthesis pathways PTA-ACKA and POXB component expression down-regulation before the overflow switch at $\mu=0.27 \pm 0.02 \mathrm{~h}^{-1}$ with concurrent 5 -fold stronger repression of acetate-consuming Acs. This in turn suggests insufficient Acs activity for consuming all the acetate produced by Pta, leading to disruption of the acetate cycling process in PTA-ACS node where constant acetyl phosphate or acetate regeneration is essential for $E$. coli chemotaxis, proteolysis, pathogenesis etc. regulation. In addition, two-substrate A-stat and D-stat experiments showed that acetate consumption capability of E. coli decreased drastically, just as Acs expression, before the start of overflow metabolism. The second step in overflow switch is the sharp decline in CAMP production at $\mu=0.45 \mathrm{~h}^{-1}$ leading to total Acs inhibition and fast accumulation of acetate.

Conclusion: This study is an example of how a systems biology approach allowed to propose a new regulation mechanism for overflow metabolism in E. coli shown by proteomic, transcriptomic and metabolomic levels coupled to two-phase acetate accumulation: acetate overflow metabolism in E. coli is triggered by Acs downregulation resulting in decreased assimilation of acetic acid produced by Pta, and disruption of the PTA-ACS node.

\section{Background}

Escherichia coli has not only been the prime organism for developing new molecular biology methods but also for producing recombinant proteins, low molecular weight compounds etc. in industrial biotechnology for decades due to its low cost manufacturing and end-

\footnotetext{
* Correspondence: raivo@kbfi.ee

'Tallinn University of Technology, Department of Chemistry, Akadeemia tee 15, 12618 Tallinn, Estonia

Full list of author information is available at the end of the article
}

product purification and its ability to reach high cell densities grown aerobically $[1,2]$. However, a major problem exists with aerobic $E$. coli cultivation on glucose at high growth rates-formation and accumulation of considerable amounts of acetic acid i.e. overflow metabolism. In addition to being detrimental for target product synthesis, accumulated acetate inhibits growth and diverts valuable carbon from biomass formation [3,4].

The acetate synthesis and utilization pathways [5] can be seen in Figure 1: acetate can be synthesized by

\section{Biomed Central}




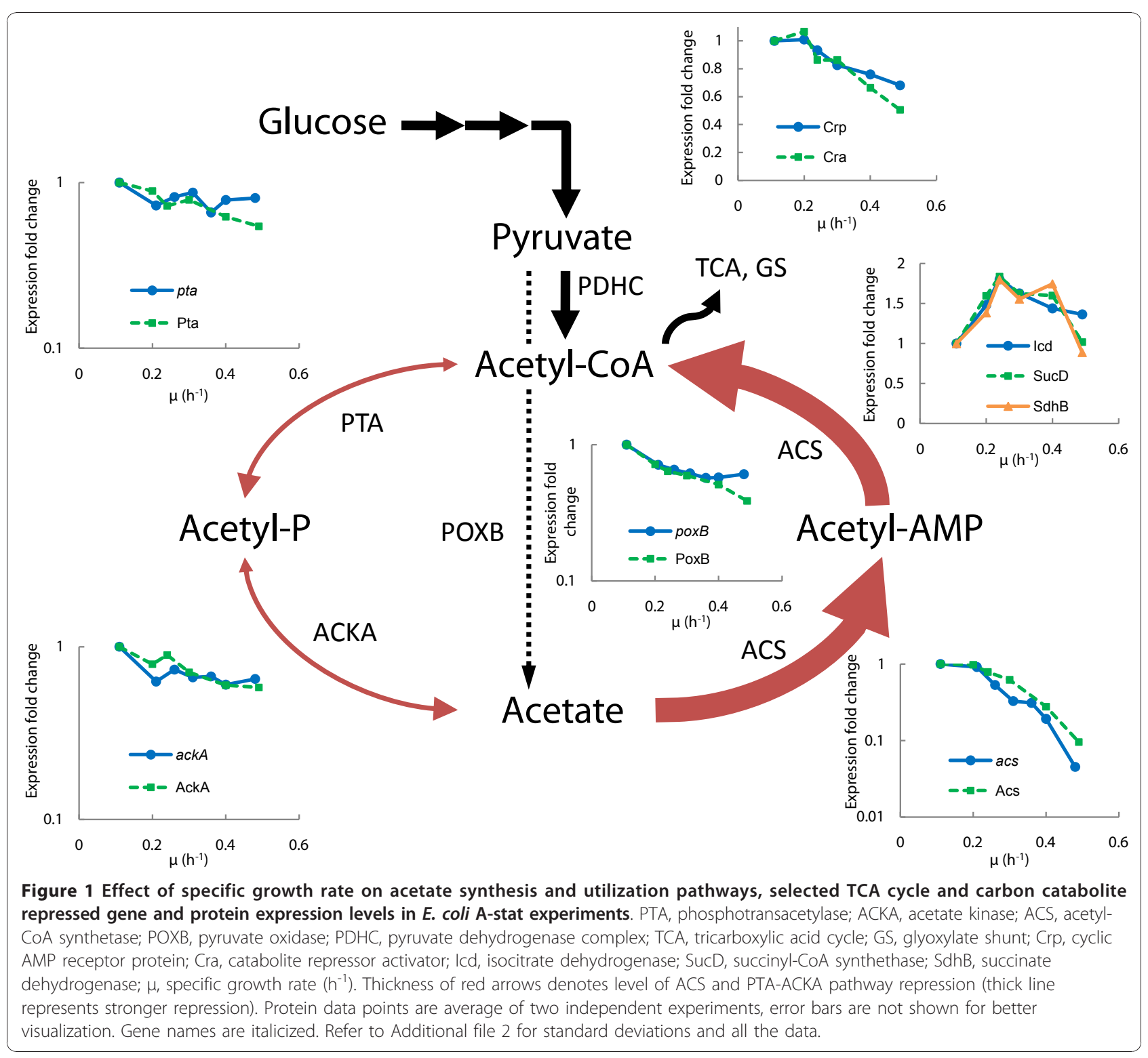

phosphotransacetylase (PTA)/acetate kinase (ACKA) and by pyruvate oxidase (POXB). Acetic acid can be metabolized to acetyl-CoA either by the PTA-ACKA pathway or by acetyl-CoA synthetase (ACS) through an intermediate acetyl-AMP. The high affinity $\left(K_{\mathrm{m}}\right.$ of 200 $\mu \mathrm{M}$ for acetic acid) ACS scavenges acetate at low concentrations whereas the low affinity PTA-ACKA pathway $\left(K_{\mathrm{m}}\right.$ of 7-10 $\left.\mathrm{mM}\right)$ is activated in the presence of high acetate concentrations [6].

The phenomenon of overflow metabolism has been studied widely over the years and it is commonly believed to be caused by an imbalance between the fluxes of glucose uptake and those for energy production and biosynthesis $[7,8]$. Several explanations such as the saturation of catalytic activities in the tricarboxylic acid (TCA) cycle $[9,10]$ and respiratory chain $[7,11,12]$, energy generation $[5,13]$ or the necessity for coenzyme A replenishment [14] have been proposed. In addition to bioprocess level approaches $[1,15]$, various genetic modifications of the acetate synthesis pathways extensively reviewed in De Mey et al. [15] have been made to minimize acetic acid production. For instance, it has been shown that deleting the main acetate synthesis route PTA-ACKA results in a strong reduction (up to $80 \%$ ) of acetate excretion, maximum growth rate ( $c a$ $20 \%$ ) and elevated levels of formate and lactate ( $c a$ 30fold) [4,16-18], whereas poxB disruption causes reduction in biomass yield ( $c$ a 25\%) and loss of aerobic growth efficiency of E. coli [19]. The latter indicates that acetate excretion cannot be simply excluded by 
disrupting its synthesis routes without encountering other unwanted effects. Unfortunately, no clear conclusions could be drawn from batch experiments with an acs knock-out strain [4]. It should be noted that studies with $E$. coli genetically modified strains engineered to diminish acetate production in batch cultures have not fully succeeded in avoiding acetate accumulation together with increasing target product production yields and rates [15]. Additionally, these studies have not allowed elucidating the mechanism of overflow metabolism unequivocally $[4,20,21]$.

Acetate overflow is a growth rate dependent phenomenon, but no study has specifically focused on growth rate dependency of protein and gene expression regulation, intra-and extracellular metabolite levels using also metabolic modeling. Describing the physiology of an organism on several 'omic levels is the basis of systems biology that facilitates better understanding of metabolic regulation [22]. In this study, E. coli metabolism at proteomic, transcriptomic and metabolomic levels was investigated using continuous cultivation methods prior to and after overflow metabolism was switched on. Usually, chemostat cultures are used for steady state metabolism analysis, however, we applied two changestat cultivation techniques: accelerostat (A-stat) and dilution rate stat (D-stat), see Methods section for details [23,24]. These cultivation methods were used as they provide three advantages over chemostat. Firstly, these changestat cultivation techniques precisely detect metabolically relevant switch points (e.g. start of overflow metabolism, maximum specific growth rate) and enable to monitor the dynamic patterns of several metabolic physiological responses simultaneously which could be left unnoticed using chemostat. Secondly, it is possible to collect vast amount of steady state comparable samples and by doing so, save time. Thirdly, both A-stat and D-stat enable to quantitatively study specific growth rate dependent coutilization of growth substrates. Latter advantage was applied for investigating acetic acid consumption capability of $E$. coli at various dilution rates in this study. Combining changestat cultivation methods enables to study metabolism responses of the same genotype at different physiological states in detail without encountering the possible metabolic artifacts accompanied when using genetically modified strains.

Results obtained by studying specific growth rate dependent changes in $E$. coli proteome, transcriptome and metabolome in continuous cultures together with metabolic modeling allowed us to propose a new theory for acetate overflow: acetate excretion in E. coli is triggered by carbon catabolite repression mediated downregulation of Acs resulting in decreased assimilation of acetate produced by Pta, and disruption of the PTAACS node.

\section{Results}

\section{E. coli metabolic switch points characterization}

In all accelerostat (A-stat) cultivation experiments, after the culture had been stabilized in chemostat at $0.10 \mathrm{~h}^{-1}$ to achieve steady state conditions, continuous increase in dilution rate with acceleration rate (a) $0.01 \mathrm{~h}^{-2}(0.01$ $\mathrm{h}^{-1}$ per hour) was started. Continuous change of specific growth rate resulted in detecting several important changes in $E$. coli metabolism as demonstrated in Figure 2. Firstly, in A-stat cultivations where glucose was the only carbon source in the medium, acetic acid started to accumulate (i.e. overflow metabolism switch) at $\mu=0.27$ $\pm 0.02 \mathrm{~h}^{-1}$ (average \pm standard deviation) and a twophase acetate accumulation pattern was observed (discussed below; Figure 2). Cells reached maximum $\mathrm{CO}_{2}$ production and $\mathrm{O}_{2}$ consumption at $\mu=0.46 \pm 0.02 \mathrm{~h}^{-1}$ and metabolic fluctuations were observed at $\mu=0.49 \pm$ $0.03 \mathrm{~h}^{-1}$ followed by washout of culture at $\mu=0.54 \pm$ $0.03 \mathrm{~h}^{-1}$ (corresponding to maximum specific growth rate at given conditions). The nature of these fluctuations will be studied further and not covered in the current publication. All A-stat results were reproduced with relative standard deviation less than $10 \%$ with the exception of acetate production per biomass ( $\left.\mathrm{Y}_{\mathrm{OAc}-}\right)$ (Table 1 and Figure S1 in Additional file 1).

\section{Metabolomic responses to rising specific growth rate}

A-stat cultivation enabled to study acetic acid accumulation profile in detail with increasing specific growth rate. Interestingly, a two-phase acetate accumulation pattern was observed (Figure 2). Slow accumulation of acetic

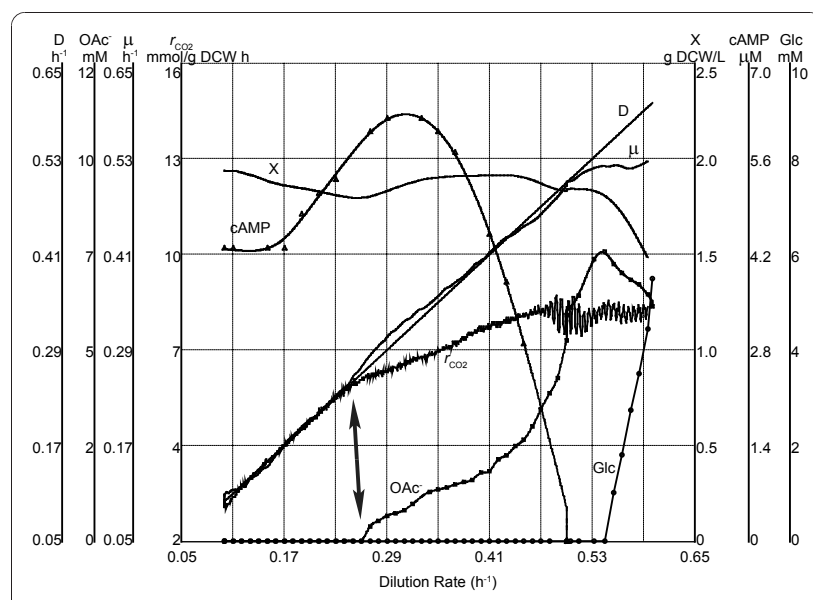

Figure 2 Increasing dilution rate dependent $E$. coli metabolism characterization in one A-stat cultivation $\left(a=0.01 \mathbf{h}^{-2}\right)$. D, dilution rate $\left(h^{-1}\right) ; X$, biomass concentration ( $g$ dry cellular weight $(\mathrm{DCW}) / \mathrm{L}) ; \mu$, specific growth rate $\left(\mathrm{h}^{-1}\right) ; r_{\mathrm{CO} 2}$, specific $\mathrm{CO}_{2}$ production rate (mmol/g DCW h); OAc; acetate concentration (mM); Glc, glucose concentration (mM); cAMP, cyclic AMP concentration $(\mu M)$. Arrow indicates the start of overflow metabolism. Start of vertical axes was chosen for better visualization. 
Table 1 A-stat and chemostat growth characteristics comparison and A-stat reproducibility over the studied specific growth rate range for three independent experiments

\begin{tabular}{|c|c|c|c|c|c|c|c|c|c|}
\hline & \multicolumn{2}{|c|}{$\mu=0.24 h^{-1}$} & \multicolumn{2}{|c|}{$\mu=0.30 \mathrm{~h}^{-1}$} & \multicolumn{2}{|c|}{$\mu=0.40 \mathrm{~h}^{-1}$} & \multicolumn{2}{|c|}{$\mu=0.51 h^{-1}$} & \multirow{2}{*}{$\begin{array}{c}\mu=0.10-0.47 \mathrm{~h}^{-1} \\
\text { A-stat RSD, \% }\end{array}$} \\
\hline & Chemostat & A-stat & Chemostat & A-stat & Chemostat & A-stat & Chemostat & A-stat & \\
\hline$Y_{X S}^{a}$ & 0.44 & $0.40 \pm 0.01$ & 0.46 & $0.41 \pm 0.01$ & 0.44 & $0.42 \pm 0.00$ & 0.43 & $0.41 \pm 0.01$ & 2.0 \\
\hline$Y_{O A C-}^{b}$ & NDE & NDE & 0.53 & $0.90 \pm 0.32$ & 1.70 & $1.56 \pm 0.23$ & 3.25 & $3.35 \pm 0.82$ & ND \\
\hline$Y_{\text {CAMP }}{ }^{\mathrm{C}}$ & 3.47 & $3.59 \pm 0.39$ & 3.25 & $3.55 \pm 0.32$ & 2.70 & $2.17 \pm 0.07$ & 0.86 & $0.71^{e}$ & 9.1 \\
\hline $\mathrm{YCO}^{\mathrm{d}}$ & 27.56 & $30.12 \pm 2.04$ & 27.55 & $27.19 \pm 1.22$ & 26.24 & $23.86 \pm 1.41$ & ND & $21.19 \pm 0.19$ & 5.6 \\
\hline
\end{tabular}

A-stat values represent the average from three independent experiments and standard deviation follows the \pm sign. Chemostat values from one experiment. NDE, not detected. ND, not determined. RSD, relative standard deviation.

${ }^{a}$ Biomass yield is given in $\mathrm{g}$ dry cell weight $(\mathrm{DCW}) / \mathrm{g}$ glucose consumed ( $\mathrm{g}$ DCW/g glucose).

${ }^{\mathrm{b}}$ Acetic acid production per biomass is given in $\mathrm{mmol}$ acetic acid/g DCW.

cAMP production per biomass is given in $\mu \mathrm{mol}$ cAMP/g DCW.

${ }^{\mathrm{d}}$ Carbon dioxide $\left(\mathrm{CO}_{2}\right)$ production per biomass is given in $\mathrm{mmol} \mathrm{CO}_{2} / \mathrm{g} \mathrm{DCW}$.

eData from one A-stat experiment.

acid started at $\mu=0.27 \pm 0.02 \mathrm{~h}^{-1}$ with concomitant change in specific $\mathrm{CO}_{2}$ production rate (Figure 2). Faster accumulation of acetate was witnessed after cells had reached maximum $\mathrm{CO}_{2}$ production at $\mu=0.46 \pm 0.02 \mathrm{~h}^{-1}$. Quite surprisingly, production of the important carbon catabolite repression (CCR) signal molecule cAMP $\left(\mathrm{Y}_{\mathrm{cAMP}}\right)$ rose from steady state chemostat level $2.45 \pm$ $0.26 \mu \mathrm{mol} / \mathrm{g}$ dry cellular weight (DCW) $\left(\mu=0.10 \mathrm{~h}^{-1}\right)$ to $3.55 \pm 0.32 \mu \mathrm{mol} / \mathrm{g}$ DCW $\left(\mu=0.30 \mathrm{~h}^{-1}\right)$ after which it sharply decreased to $1.30 \pm 0.44 \mu \mathrm{mol} / \mathrm{g} \mathrm{DCW}$ at $\mu=0.45$ $\mathrm{h}^{-1}$ (Figure S1 in Additional file 1). This abrupt decline took place simultaneously with the faster acetate accumulation profile described above (Figure 2 and Figure S1 in Additional file 1). In addition, similar two-phase acetate accumulation phenomenon was observed in a twosubstrate (glucose + acetic acid) A-stat during the decrease of cAMP around specific growth rate $0.39 \mathrm{~h}^{-1}$ (Figure S2 in Additional file 1).

Significant fall in two of the measured pentose phosphate pathway intermediates ribose-5-phosphate (R5P) and erythrose-4-phosphate (E4P) was detected with increasing specific growth rate which could point to possible limitation in RNA biosynthesis during growth (Figure 3A). PTA-ACS node related compound nonesterified acetyl-CoA (HS-CoA) level declined two-fold simultaneously with cAMP after acetate started to accumulate (Figure $3 \mathrm{~B}$ ). This indicates the possible increase of other CoA containing compounds e.g. succinyl-CoA. Accumulation of TCA cycle intermediates $\alpha$-ketoglutarate and isocitrate (Figure $3 \mathrm{~B}$ ) with increasing dilution rate could be associated with pyrimidine deficiency and decrease of ATP expenditure in the PTA-ACS cycle. Concurrently, intracellular concentrations of fructose-1,6-bisphosphate (FBP) and glyceraldehyde-3-phosphate (GAP) from the upper part of energy generating glycolysis increased 6- and 3-fold, respectively (Figure 3C).

\section{Functional-genomic responses to rising specific growth} rate

The two main known pathways for acetate synthesis phosphotransacetylase-acetate kinase (PTA-ACKA) and pyruvate oxidase (POXB) were down-regulated, both on gene and protein expression levels, from $\mu=0.20$ $\mathrm{h}^{-1}$ i.e. before acetate overflow was switched on. At the same time, there was a concurrent 10 -fold repression of the acetic acid utilization enzyme acetyl-CoA synthetase (Acs). This substantial difference (5-fold) between the acetate synthesis and assimilation pathways expression suggests that the synthesized acetic acid cannot be fully assimilated with increasing growth rates (Figure 1).

We observed the beginning of carbon catabolite repression (CCR) induction prior to acetate accumulation in parallel with Acs down-regulation. This was indicated by down-regulation (3-fold on average) of CCRmediated components: alternative (to glucose) substrate transport and utilization systems like galactose (MglAB), maltose (MalBEFKM), galactitol (GatABC), L-arabinose (AraF), D-ribose (RbsAB), $\mathrm{C}_{4}$-dicarboxylates (DctA) and acetate (ActP, YjcH) (Figure $4 \mathrm{C}$ and Additional file 2). Moreover, expression of transcription activator Crp (cyclic AMP receptor protein which regulates the expression of Acs transcribing acs-yjcH-actP operon) and Cra (catabolite repressor activator; a global transcriptional protein essential for acetic acid uptake [25]) were reduced 1.5 and 2 times, respectively, in like manner to carbon catabolite repressed proteins mentioned above (Figure 1). Simultaneously, components of the gluconeogenesis pathway (Pck, MaeB, Pps) and glyoxylate shunt enzymes AceA, AceB (vital for acetate consumption) were repressed with growth rate increase (Figure 4B and Additional file 2). It should be emphasized that most of the TCA cycle gene and protein levels were maintained or even increased up to $\mu=0.40 \mathrm{~h}^{-1}$ 

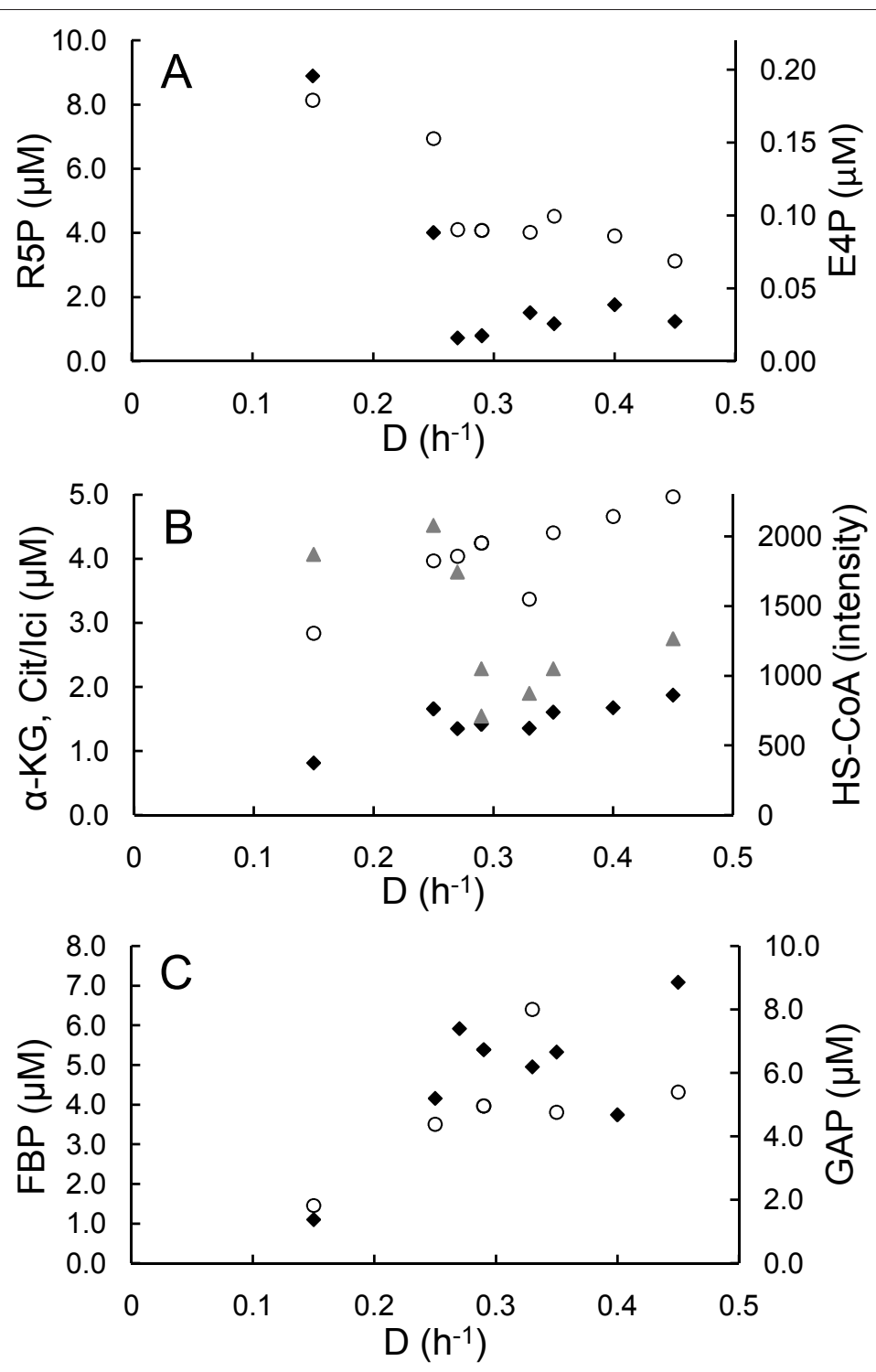

Figure 3 Dilution rate dependent intracellular metabolite patterns in one $E$. coli A-stat experiment. D, dilution rate $\left(h^{-1}\right)$. (A) Pentose phosphate pathway metabolites. R5P, ribose-5-phosphate concentration (black diamond); E4P, erythrose-4-phosphate concentration (open circle). (B) TCA cycle metabolites and co-factor free CoA. $\alpha-K G, \alpha$-ketoglutarate concentration (black diamond); Cit/lci, citrate/isocitrate pool concentration (open circle); HS-CoA, co-factor free CoA level (grey triangle). (C) Glycolysis (upper part) metabolites. FBP, fructose-1,6-bisphosphate concentration (black diamond); GAP, glyceraldehyde-3-phosphate concentration (open circle).

followed by sudden repression simultaneous to achieving maximum specific $\mathrm{CO}_{2}$ production rate $(\mu=0.46 \pm$ $0.02 \mathrm{~h}^{-1}$, see above; Figure 1 Figure 2 and Figure 4A). This may allude to no limitation at the TCA cycle level around the specific growth rate where overflow metabolism was switched on.

\section{Acetic acid consumption capability studied by dilution} rate stat (D-stat) and two-substrate A-stat cultivations The beginning of a strong decrease in acetate assimilation enzyme Acs expression before overflow switch point implies to a possible connection between acetate assimilation capability and overflow metabolism of acetate (Figure 1). Therefore, specific growth rate dependent acetic acid consumption capabilities were investigated using D-stat and two-substrate A-stat methods. It was shown by $\mathrm{D}$-stat experiments at various dilution rates that more than a 12 -fold reduction in acetate consumption capability took place around overflow switch point, and its total loss was detected between dilution rates 0.45 and $0.505 \pm 0.005 \mathrm{~h}^{-1}$ (Figure 5). Acetic acid consumption and production was also studied in a single experiment using two substrate (glucose + acetic acid) A-stat cultivation (Figure S2 in Additional file 1) which 

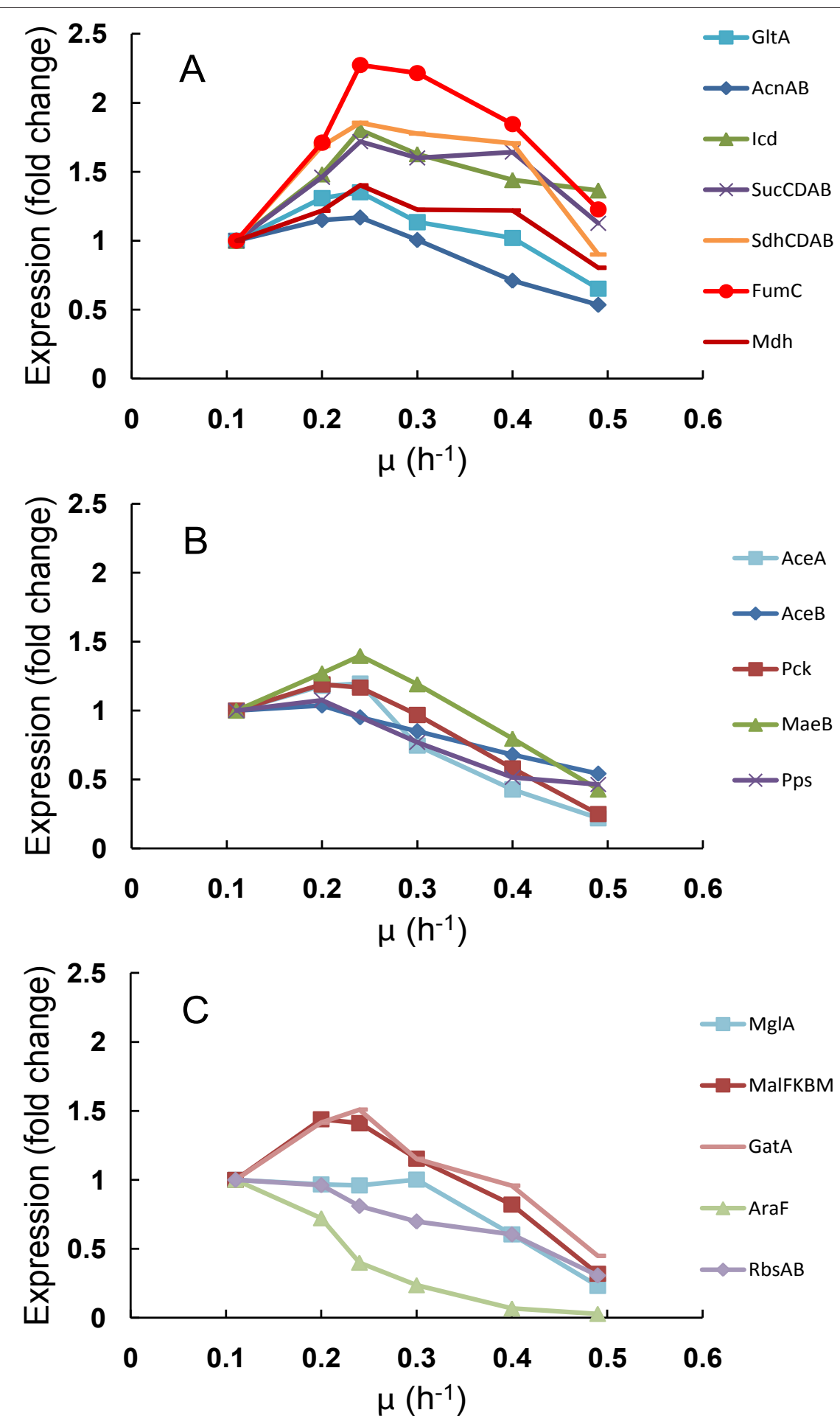

Figure 4 Specific growth rate dependent TCA cycle, glyoxylate shunt, glyconeogenesis and carbon catabolite repressed protein expression changes in $E$ coli A-stat cultures. $\mu$, specific growth rate $\left(h^{-1}\right)$. (A) TCA cycle (average of proteins from the same operon are depicted as one point e.g. AcnAB). (B) Glyoxylate shunt (AceA, AceB) and glyconeogenesis. (C) Carbon catabolite repressed proteins. Protein data points are average of two independent experiments, error bars are not shown for better visualization (refer to Additional file 2 for standard deviations). 


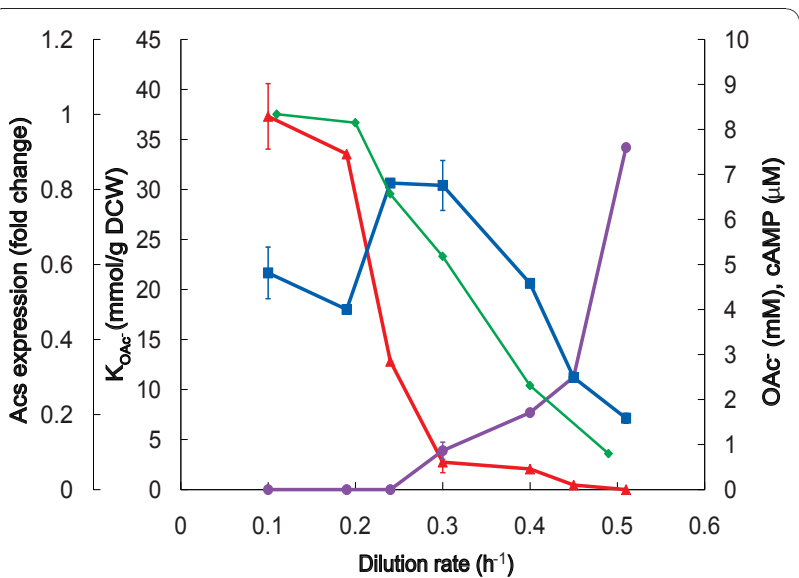

Figure 5 Dilution rate dependent acetic acid consumption

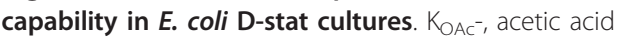
consumption per biomass (red triangle); OAc, acetate concentration in chemostat before the start of acetic acid supplemented medium addition (violet circle); cAMP, cyclic AMP concentration (blue square); Acs, acetyl-CoA synthetase protein dilution rate dependent expression levels from A-stat (green diamond). Error bars represent the standard deviation from two independent D-stat experiments.

demonstrated that acetic acid consumption started to decrease at $\mu=0.25 \mathrm{~h}^{-1}$ and was completely abolished at $\mu=0.48 \mathrm{~h}^{-1}$ which fits into the range of dilution rates observed in D-stat.

\section{A-stat comparison with chemostat}

As could be seen from Table 1 major growth characteristics such as biomass yield $\left(\mathrm{Y}_{\mathrm{XS}}\right)$, acetate $\left(\mathrm{Y}_{\mathrm{OAc}-}\right)$, cyclic AMP $\left(\mathrm{Y}_{\mathrm{CAMP}}\right)$ and carbon dioxide $\left(\mathrm{Y}_{\mathrm{CO} 2}\right)$ production per biomass from A-stat and chemostat are all fully quantitatively comparable. The latter results enable to use Astat data for quantitative modeling calculations. In addition, the two continuous cultivation methods were examined at transcriptome level using DNA microarrays. Transcript spot intensities from quasi steady state A-stat sample at $\mu=0.48 \mathrm{~h}^{-1}$ and chemostat sample at $\mu=0.51 \mathrm{~h}^{-1}$ showed an excellent Pearson productmoment correlation coefficient $\mathrm{R}=0.964$ (Figure S3 in Additional file 1; Additional file 3). This indicates good biological correlation between $E$. coli transcript profiles at similar specific growth rates in chemostat and A-stat. These results showed that our quasi steady state data from A-stat and D-stat cultures are steady state representative.

\section{Proteome and transcriptome comparison}

E. coli protein expression ratios for around 1600 proteins were generated by comparing two biological replicates at specific growth rates $0.20 \pm 0.01 ; 0.26 ; 0.30 \pm$ $0.01 ; 0.40 \pm 0.00 ; 0.49 \pm 0.01 \mathrm{~h}^{-1}$ with sample at $\mu=$ $0.10 \pm 0.01 \mathrm{~h}^{-1}$ (chemostat point prior to the start of acceleration in A-stat) which produced Pearson correlation coefficients for two biological replicates in the indicated pairs of comparison in the range of $\mathrm{R}=0.788$ 0.917 (Figure S4 in Additional file 1).

DNA microarray analysis of 4,321 transcripts was conducted with the Agilent platform using the samples from one A-stat cultivation. Gene expression ratios between specific growth rates $0.21 ; 0.26 ; 0.31 ; 0.36 ; 0.40$; $0.48 \mathrm{~h}^{-1}$ and $\mu=0.11 \mathrm{~h}^{-1}$ (chemostat point prior to the start of acceleration in A-stat) were calculated. Comparison of gene and protein expression changes (between respective specific growth rates) revealed that components of the PTA-ACS node were regulated at transcriptional level as the absolute majority of the studied transcripts and proteins indicated by the good correlation between transcriptome and proteome expression profiles (Figure 1 and Figure S5 in Additional file 1).

Most recent studies have either failed to find a significant correlation between protein and mRNA abundances or have observed only a weak correlation (reviewed in [22]). It has been suggested that the main reasons for uncoupling of mRNA and protein abundances are protein regulation by post-translational modification, post-transcriptional regulation of protein synthesis, differences in the half-lives of mRNA and proteins, or possible functional requirement for protein binding [22]. As the cells in these studies were mostly cultured in non steady state condition, our steady state data with very good correlation between transcriptome and proteome implies that the physiological state of the culture (steady state vs. non steady state) could be an important factor in terms of mRNA and protein correlation determination. Transcriptome and proteome data are presented in Additional file 2 and at NCBI Gene Expression Omnibus and PRIDE database (see Methods for details), respectively.

\section{Discussion}

To gain better insights into the regulation of acetate overflow metabolism in $E$. coli, we studied specific growth rate dependent proteomic, transcriptomic and metabolomic patterns combined with metabolic modeling using advanced continuous cultivation methods, which has not been carried out before. Continuous monitoring of the specific growth rate effect on $E$. coli metabolism enabled us to precisely detect important metabolic shift points, the most important being the start of acetate overflow at $\mu=0.27 \pm 0.02 \mathrm{~h}^{-1}$ (Figure 2 ), and changing patterns of a number of important metabolites e.g. acetate, cAMP. Quite surprising was the down-regulation of the known acetate synthesis pathways, PTA-ACKA and POXB expression before overflow switch with increasing growth rate (Figure 1). A similar pattern has been seen before in chemostat cultures 
but without emphasizing the possible physiological consequences [26-28]. A 10-fold repression of the acetic acid utilization enzyme acetyl-CoA synthetase (Acs) expression was observed concurrently with the downregulation of the PTA-ACKA pathway indicating that acetic acid synthesis might exceed its assimilation (Figure 1). Our two substrate A-stat and D-stat experiments directly proved that acetate consumption capability of $E$. coli is specific growth rate dependent as acetate consumption started to decrease at $\mu=0.25 \mathrm{~h}^{-1}$ (Figure S2 in Additional file 1) and acetate consumption capability decreased 12-fold around overflow switch growth rate $\mu=0.27 \pm 0.02 \mathrm{~h}^{-1}$, respectively (Figure 5). In addition, it was shown that activation of carbon catabolite repression (CCR) and repression of Acs take place simultaneously prior to the start of overflow metabolism (Figure 1 Figure 4 and Figure 5). As a result, it is proposed that acetate overflow metabolism in E. coli is triggered by Acs down-regulation resulting in decreased assimilation of acetic acid produced by Pta, and disruption of the PTA-ACS node.

We showed that Acs was concurrently down-regulated five times more compared to the acetate synthesis pathways (Figure 1). In addition, the TCA cycle flux decrease as shown by change in $\mathrm{CO}_{2}$ production at overflow switch growth rate indicates that carbon is not metabolized by the TCA cycle after the start of acetate accumulation with pre overflow switch rates (Figure 2 and Additional file 4). The latter is caused because the amount of acetyl-CoA entering the TCA cycle decreases after carbon is lost into excreted acetate. Stronger repression of the acetate consuming Acs in comparison with acetate synthesizing PTA-ACKA together with a decline in TCA cycle flux suggest disruption of acetic acid cycling at the PTA-ACS node (Figure 1). While this node may seem as a futile cycle, the fact is that numerous metabolic tasks involving the intermediate molecules of this cycle-acetyl phosphate (acetyl-P) and acetyl-AMP-are essential for proper E. coli growth (Figure 6). For instance, these molecules play a crucial role in bacterial chemotaxis regulation in which flagellar rotation is controlled by the activation level of the response regulator CheY [29] through either phosphotransfer from CheA [30,31] or acetyl-P [31,32], acetylation by acetyl-AMP $[33,34]$ or co-regulation of both mechanisms [29]. It has been also demonstrated that acetyl-P synthesis is vital for EnvZ-independent regulation of outer membrane porins [35], pathogenesis [36] and regulation of several virulence factors [5]. Furthermore, it has been presented that acetyl-P interacts with phosphate concentration regulators PhoB-PhoR [37] and NRI protein which is part of a complex nitrogen sensing system [38]. Acetyl-P is critical for efficient degradation of unfolded or damaged proteins by ATP-dependent

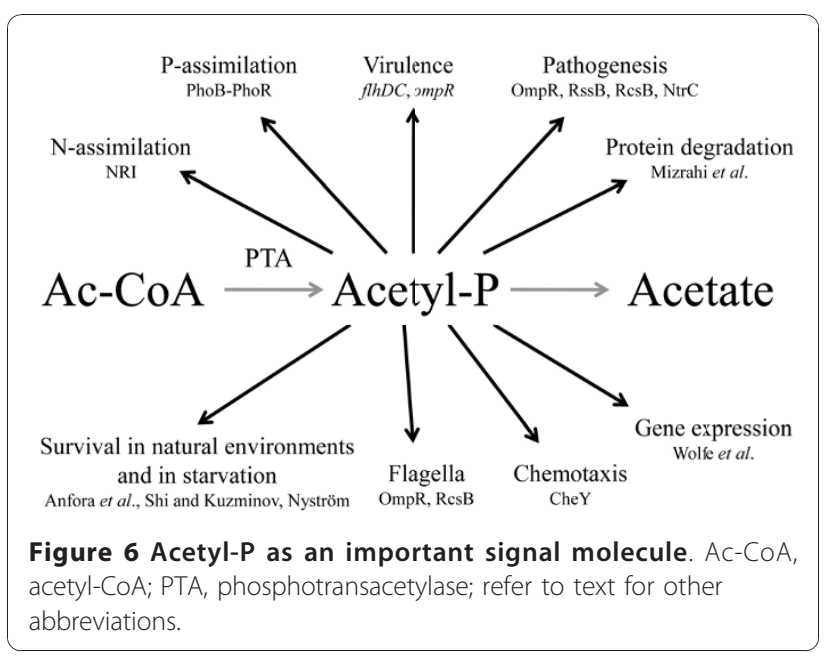

proteases [39]. Altogether, acetyl-P can influence the regulation of almost 100 other genes [40]. Finally, pta and/or ackA mutations were shown to affect repair-deficient E. coli mutants [41] and a pta mutant has been reported to be impaired in its ability to survive during glucose starvation, while the ack $A$ mutant survived as well as the parent strain [42]. It is important to note that the only known pathway in E. coli for acetyl-P synthesis is the PTA-ACKA [5,31]. Taking all the previous into account, we conclude that acetyl-P as well as acetyl-AMP are essential for cellular growth of E. coli, and as acetic acid formation is the result of their dephosphorylation, acetic acid should be synthesized and consumed simultaneously during growth to maintain proper balance between metabolites of the PTA-ACS node. This is in agreement with Shin et al. [28] who proposed that wild-type $E$. coli constitutively synthesizes acetate even when growing on non-acetogenic carbon source succinate or at low growth rates in carbon limited cultures. It also has to be mentioned that acetic acid is a by-product in the synthesis of cysteine, methionine and arginine, covering around $0.4 \mathrm{mmol} / \mathrm{g} \mathrm{DCW}$ (Additional file 4). Based on our experimental and literature data, production and re-assimilation of acetate might be over $1 \mathrm{mmol} / \mathrm{g} \mathrm{DCW}$ at $\mu=0.2 \mathrm{~h}^{-1}$ (Text S2 in Additional file 1) which further supports the hypothesis of the necessity for constant acetic acid synthesis.

A similar regulation for overflow metabolism of acetate was posed for Saccharomyces cerevisiae by Postma and co-workers: they postulated that acetate accumulation is the result of insufficient acetyl-CoA synthetase activity for the complete functioning of the pyruvate dehydrogenase bypass because of glucose repression of ACS at high growth rates [43]. The hypothesis proposed here is also consistent with the observation that an acs mutant of $E$. coli accumulated acetate in chemostat cultures at dilution rate (D) $0.22 \mathrm{~h}^{-1}$ whereas acetate 
overflow was started in wild-type at a higher $\mathrm{D}=0.35 \mathrm{~h}^{-1}$ [28]. Furthermore, it has been shown that over-expression of acs [44] and constitutively expressed acs together with glyoxylate shunt repressors $i c l R$ and $f a d R$ mutant resulted in a significant reduction in acetate accumulation in glucose batch fermentations [28]. Adams and co-workers showed that as a result of micro-evolution, E. coli increased acetate consumption capability by over-expressing Acs (not AckA) [45,46], further supporting the connection between Acs activity and acetate accumulation.

As Acs down-regulation is responsible for triggering overflow metabolism and the resulting accumulation of acetate is detrimental to cellular growth, it bears questioning why E. coli has not evolved towards maintaining sufficient Acs levels for acetate assimilation in all growth conditions. Growth conditions in E. coli native environments are rough as concentrations of utilizable carbon sources including acetate are in the low $\mathrm{mg} \mathrm{L}^{-1}$ range and access to nutrients is troublesome [47]. These harsh conditions force $E$. coli to make its metabolism ready for scavenging all possible carbon sources including acetate. However, in nutrient rich laboratory conditions, E. coli focuses on anthropic growth [48] and biomass production rate, primarily realized by enhancing readily oxidizable substrate (glucose) uptake kinetics which in turn results in Acs repression through CCR and thus, acetate accumulation [46]. This indicates that an active Acs is not essential for rapid growth for E. coli. It seems that maintaining high expression levels of acetate assimilation components (and also other alternative substrates ones) is energetically not favorable at higher growth rates. Moreover, as the space on cell membrane is limited and as E. coli achieves more rapid growth probably by increasing the number of glucose transport machinery components on the membrane, using area for alternative substrate transport proteins is not beneficial for faster growth. Interestingly, even in one of its natural environments-urinary tract-where a continuous dilution of acetate occurs, it has been shown that metabolizing acetate to acetyl-CoA by Acs is not essential for normal E. coli colonization as PTA-ACKA pathway and maintenance of a proper intracellular acetyl-P concentration are necessary for colonizing murine urinary tract [32].

Based on all the points discussed above, PTA-ACS might function as a futile cycle to provide rapid regulation of acetyl-P concentration in the cell for an active chemotaxis that is vital in natural nutrient-depleted environments, fighting against other organisms (acetate production), pathogenesis, biofilm formation etc. This hypothesis is consistent with the fact that the flagellar assembly and regulation operon (tar-tap-cheRBYZ) was more intensively expressed at lower growth rates (Additional file 2) where residual glucose concentration is smaller.
Concerning Acs down-regulation, it is possible that CCR is responsible for its repression as proposed by Treves et al. [46] showing the link between ACS expression level and acetate accumulation. In our experiments, it was shown that activation of CCR and repression of Acs take place simultaneously prior to the start of overflow metabolism (Figure 1 and Figure 4). As it is well known that CCR is initiated by the presence of glucose in the medium $[49,50]$, we propose that increasing residual glucose concentration accompanying smooth rise of dilution rate in A-stat triggers Acs down-regulation by CCR. The cAMP-Crp complex is one of the major players in CCR of E. coli as cAMP binding to Crp drastically increases its affinity towards activating the promotors of catabolic enzymes, including Acs $[6,49,50]$. We measured a 1.5-fold decrease in Crp expression with increasing growth rate (Figure 1) that is in agreement with the data in the literature [51]. In addition, when $E$. coli mutant defective in the gene crp was cultivated in glucose-limited chemostat at a low $\mathrm{D}=0.10 \mathrm{~h}^{-1}$, it accumulated acetate whereas the wild-type did not [52]. Furthermore, it exhibited a 34\% higher biomass yield relative to the wild-type-this increase might be explained by reduced ATP wasting in the acetate futile cycle, which can be directed to biomass synthesis. Moreover, Khankal et al. [53] noted that E. coli CRP* mutants that do not require Crp binding to cAMP to activate the expression of catabolic genes showed lowered glucose effect on xylose consumption, 3.6 times higher acs expression levels and secreted substantially less acetate in xylitol producing batch fermentations. The connection between cAMP concentration and acetic acid consumption capability, together with the two-phase acetate accumulation profile observed in A-stat and D-stat cultures (Figure 2 and Figure 5) suggests a correlation between increasing residual glucose concentration mediated cAMP-Crp repression and acetate accumulation. Thus, cAMP-Crp dependent regulation of Acs transcribing acs-yjcH-actP operon might be a reason for acetate excretion, as also proposed by Veit et al. [10]. Our hypothesis of the CCR mediated acetate overflow metabolism is as well in agreement with the fact that rising glucose lowers the intracellular Crp level through the autoregulatory loop of the crp gene [54]. However, other mechanisms can also be involved in Acs downregulation, for example by Cra (Figure 1). Indeed, Sarkar and colleagues have shown that glucose uptake and acetate production rates increased with a decrease of acetate consumption in an E. coli cra mutant [55].

What could be the biological relevance of the disruption of the PTA-ACS node? Firstly, decline of the ATPspending PTA-ACS cycle throughput with increasing growth rate points to possible lower ATP spilling (our model calculations). Secondly, disruption of the PTA- 
ACS node decreases the energy needed for expression of this cycle's components. As the disruption of PTA-ACS cycle is CCR-mediated, repression of other alternative substrate transport and utilization enzymes by CCR enables to save additional energy. This could all lead to the decrease of ATP production as was indicated by the diminishing TCA cycle fluxes (Figure 2). Hence, it is plausible that cells repress (by CCR) the expression levels of alternative substrate utilization components (including Acs) for making space on the cell membrane for more preferred substrate (glucose) utilization and ATP producing components to achieve faster growth (see above).

Finally, it was demonstrated that highly reproducible A-stat data are well comparable to chemostat at the level of major growth characteristics and transcriptome, hence quasi steady state data from A-stat can be considered steady state representative (Table 1; Figure S1 and Figure S3 in Additional file 1). Furthermore, as shown also by Postma et al. for S. cerevisiae [43], chemostat is not fully suitable for characterization of dilution rate dependent metabolic transitions, whereas A-stat should be considered an appropriate tool for this. A-stat is especially well suited for the studies of the details of transient metabolism processes. Dynamic behavior of acetate, cAMP etc. with increasing specific growth rate (Figure 2 Figure 3 and Figure S1 in Additional file 1) and change in acetic acid consumption capability in the two-substrate A-stat (Figure S2 in Additional file 1) could be cited as good examples of the latter.

\section{Conclusion}

This study is an excellent example of how a systems biology approach using highly reproducible advanced cultivation methods coupled with multiple 'omics analysis and metabolic modeling allowed to propose a new possible regulation mechanism for overflow metabolism in E. coli: acetate overflow is triggered by carbon catabolite repression mediated Acs down-regulation resulting in decreased assimilation of acetate produced by Pta, and disruption of the PTA-ACS node. The practical implications derived from this could lead to better engineering of $E$. coli in overcoming several metabolic obstacles, increasing production yields etc.

\section{Methods \\ Bacterial strain, medium and continuous cultivation conditions}

The E. coli K12 MG1655 ( $\lambda^{-} \mathrm{F}^{-} r p h-1 F n r^{+}$; Deutsche Sammlung von Mikroorganismen und Zellkulturen, Germany) strain was used in all experiments. Growth and physiological characteristics in accelerostat (A-stat) cultivations were determined using a defined minimal medium as described before by Nahku et al. [51], except
$4.5 \mathrm{~g} / \mathrm{L} \alpha$-(D)-glucose and $100 \mu \mathrm{L} \mathrm{L}^{-1}$ Antifoam C (Sigma Aldrich, St. Louis, LO) was used. The latter was also used in dilution rate stat (D-stat) experiments as the main cultivation medium. In addition, a second medium was used in D-stat where the main medium was supplemented by acetic acid and prepared as follows: $300 \mathrm{ml}$ medium was withdrawn from the main cultivation medium and supplemented with $3 \mathrm{ml}$ of glacial acetic acid (99.9\%). One A-stat experiment (referred to as two-substrate A-stat) was carried out with the same medium as other A-stats, but in addition supplemented with acetic acid (final concentration $5 \mathrm{mM}$ ).

The continuous (both A-stat and D-stat) cultivation system consisted of 1.25 L Biobundle bioreactor (Applikon Biotechnology B.V., Schiedam, the Netherlands) controlled by an ADI 1030 biocontroller (Applikon Biotechnology B.V.) and a cultivation control program "BioXpert NT" (Applikon Biotechnology B.V.). The system was equipped with $\mathrm{OD}, \mathrm{pH}, \mathrm{pO}_{2}, \mathrm{CO}_{2}$ and temperature sensors. The bioreactor was set on a balance whose output was used as the control variable to ensure constant culture volume $(300 \pm 1 \mathrm{~mL})$. Similarly, the inflow was controlled through measuring the mass of the fresh culture medium.

A-stat cultivation system and control algorithms used are described in more detail in our previous works $[24,51,56]$. Dilution rate stat (D-stat) is a continuous cultivation method where dilution rate is constant as in a chemostat while an environmental parameter is smoothly changed [24]. The D-stat experiments in this study were carried out with a slight modification: instead of changing an environmental parameter, two different media were used to keep dilution rate constant. After achieving steady state conditions in chemostat using minimal medium supplemented with glucose, addition of the second medium complemented with glucose and acetic acid was started. The feeding rate of the initial medium was decreased at the same time, resulting in constant glucose concentration in the feed. The acetic acid concentration in the bioreactor as a result of inflow has to be determined to enable precise acetic acid consumption/production rate calculation for the bacteria. Hence, increase of acetic acid concentration in bioreactor was calculated and validated in duplicate noninoculatedD-stat test experiments producing an average standard deviation of $1.24 \mathrm{mM}$ between calculated and measured acetic acid concentrations.

All continuous cultivation experiments were carried out at $37^{\circ} \mathrm{C}, \mathrm{pH} 7$ and under aerobic conditions (air flow rate $150 \mathrm{ml} \mathrm{min}^{-1}$ ) with an agitation speed of 800 rpm. Four A-stat cultivations were performed with acceleration rate (a) $0.01 \mathrm{~h}^{-2}$. Duplicate D-stat experiments were performed at dilution rates 0.10 ; $0.30 ; 0.505$ $\pm 0.005 \mathrm{~h}^{-1}$ and single experiments at $0.19 ; 0.24$; 
$0.40 ; 0.45 \mathrm{~h}^{-1}$. The acetic acid addition profile was set to achieve $32 \pm 6 \mathrm{mM}$ and $58 \pm 5 \mathrm{mM}$ in 7 hours inside the bioreactor for experiments at dilution rates 0.10$0.24 \mathrm{~h}^{-1}$ and $0.30-0.51 \mathrm{~h}^{-1}$, respectively. The growth characteristics of the bacteria were calculated on the basis of total volume of medium pumped out from bioreactor (L), biomass (g DCW), organic acid concentrations in culture medium $(\mathrm{mM})$ and $\mathrm{CO}_{2}$ concentration in the outflow gas (mM). Formulas were as described in a previous study [24]. It should be noted that the absolute $\mathrm{CO}_{2}$ concentrations could be error-prone due to measurement difficulties. However, this does not influence the dynamic pattern of specific $\mathrm{CO}_{2}$ production rate $\left(r_{\mathrm{CO} 2}\right)$ during specific growth rate increase.

\section{Analytical methods}

The concentrations of organic acids (lactate, acetate and formate), ethanol and glucose in the culture medium were determined by HPLC and cellular dry weight (expressed as DCW) as described by Nahku et al. [51].

\section{Protein expression analysis}

Refer to Text S1 in Additional file 1 for detailed description. Shortly, protein expression ratios for around 1600 proteins (identified for each growth rate at a $>95 \%$ confidence interval in average from 89,303 distinct 2 or more high-confidence peptides) were generated from mass spectrometric spectra by firstly calculating the ratios between continuous cultivation samples at specific growth rates $0.10 \pm 0.01 \mathrm{~h}^{-1}$ (chemostat point prior to the start of acceleration in A-stat); $0.20 \pm 0.01 ; 0.26 ; 0.30 \pm 0.01 ; 0.40 \pm 0.00 ; 0.49 \pm$ $0.01 \mathrm{~h}^{-1}$ and batch sample grown on medium containing ${ }^{15} \mathrm{NH}_{4} \mathrm{Cl}$ as the only source of ammonia. Secondly, the ratios between the mentioned specific growth rates with chemostat point $\left(\mu=0.10 \pm 0.01 \mathrm{~h}^{-1}\right)$ for two biological replicates were calculated to yield protein expression levels for respective specific growth rates. Protein (and gene) expression measurement results are shown in Additional file 2. Proteomic analysis data is also available at the PRIDE database [57]http://www.ebi.ac.uk/ pride under accession numbers 12189-12199 (username: review74613, password: Ge9T48e8). The data was converted using PRIDE Converter http://code.google.com/ $\mathrm{p} /$ pride-converter [58].

\section{Gene expression profiling}

DNA microarray analysis of 4,321 transcripts was conducted with the Agilent platform using the data from one A-stat cultivation $\left(\mathrm{a}=0.01 \mathrm{~h}^{-2}\right)$, and gene expression ratios between specific growth rates $0.21 ; 0.26 ; 0.31$; $0.36 ; 0.40 ; 0.48 \mathrm{~h}^{-1}$ and $\mu=0.11 \mathrm{~h}^{-1}$ were calculated. Transcript spot intensities of chemostat sample (sample from D-stat prior to acetic acid addition) from $\mu=0.51$ $\mathrm{h}^{-1}$ and A-stat $\mu=0.48 \mathrm{~h}^{-1}$ were used for the two method's comparison at transcriptome level. Gene (and protein) expression measurement results are shown in Additional file 2. DNA microarray data is also available at NCBI Gene Expression Omnibus (Reference series: GSE23920). The details of the procedure are provided in Text $\mathrm{S} 1$ in Additional file 1.

\section{Metabolome analysis}

Sampling was carried out by the rapid centrifugation method. Acquity UPLC (Waters, Milford, MA) together with end-capped HSS C18 T3 $1.8 \mu \mathrm{m}, 2.1 \times 100 \mathrm{~mm}$ column for compound separation coupled to TOF-MS with an electrospray ionization (ESI) source was used for detection (LCT Premiere, Waters). The details of the procedure are provided in Text S1 in Additional file 1.

\section{Additional material}

Additional file 1: Detailed Methods (Text S1); calculation of acetate reconsumption (Text S2); Supplementary Figures S1-S5.

Additional file 2: Growth rate dependent gene (one A-stat) and average protein expression changes of two A-stat experiments with Escherichia coli K12 MG1655. Transcriptome and proteome analysis results, also with standard deviations.

Additional file 3: Gene spot intensities of A-stat at $\mu=0.48 \mathrm{~h}^{-1}$ and chemostat at $\mu=0.51 \mathrm{~h}^{-1}$ experiments with Escherichia coli K12 MG1655. Data for A-stat and chemostat transcriptome comparison.

Additional file 4: Simplified metabolic flux analysis. Detailed description of model calculations with simplified metabolic flux analysis.

\section{Acknowledgements}

The financial support for this research was provided by the Enterprise Estonia project EU29994, and Ministry of Education, Estonia, through the grant SF0140090s08. The authors would like to thank Lauri Peil and Elina Pelonen for help in carrying out 'omics analysis.

\section{Author details}

${ }^{1}$ Tallinn University of Technology, Department of Chemistry, Akadeemia tee 15, 12618 Tallinn, Estonia. ${ }^{2}$ Competence Centre of Food and Fermentation Technologies, Akadeemia tee 15b, 12618 Tallinn, Estonia. ${ }^{3}$ Tallinn University of Technology, Department of Food Processing, Ehitajate tee 5, 19086 Tallinn, Estonia.

\section{Authors' contributions}

$\mathrm{KV}, \mathrm{KA}$, and RV drafted the manuscript. $\mathrm{RN}, \mathrm{PL}$, and $\mathrm{LA}$ helped in preparing the manuscript. KV, RN, and PL designed and performed the experiments. $\mathrm{KV}$ analysed the experimental data. RN, PL, and LA carried out the 'omics analysis. KV, KA, and RV guided and coordinated the project. All authors read and approved the manuscript.

Received: 16 June 2010 Accepted: 1 December 2010 Published: 1 December 2010

\section{References}

1. Eiteman MA, Altman E: Overcoming acetate in Escherichia coli recombinant protein fermentations. Trend Biotechnol 2006, 24:530-536.

2. Clomburg JM, Gonzalez R: Biofuel production in Escherichia coli: the role of metabolic engineering and synthetic biology. Appl Microbiol Biotechnol 2010, 86:419-434. 
3. Nakano K, Rischke M, Sato S, Märkl H: Influence of acetic acid on the growth of Escherichia coli K12 during high-cell-density cultivation in a dialysis reactor. Appl Microbiol Biotechnol 1997, 48:597-601.

4. Contiero J, Beatty CM, Kumari S, DeSanti CL, Strohl WR, Wolfe AJ: Effects of mutations in acetate metabolism in high-cell-density growth of Escherichia coli. J Ind Microbiol Biotechnol 2000, 24:421-430.

5. Wolfe AJ: The acetate switch. Microbiol Mol Biol Rev 2005, 69:12-50.

6. Kumari S, Beatty CM, Browning DF, Busby SJ, Simel EJ, Hovel-Miner G, Wolfe AJ: Regulation of acetyl coenzyme A synthetase in Escherichia coli. J Bacteriol 2000, 182:4173-4179.

7. Han K, Lim HC, Hong J: Acetic acid formation in Escherichia coli fermentation. Biotechnol Bioeng 1992, 39:663-671.

8. Farmer WR, Liao JC: Reduction of aerobic acetate production by Escherichia coli. Appl Environ Microbiol 1997, 63:3205-3210.

9. Majewski RA, Domach MM: Simple constrained-optimization view of acetate overflow in E. coli. Biotechnol Bioeng 1990, 35:732-738.

10. Veit A, Polen $T$, Wendisch V: Global gene expression analysis of glucose overflow metabolism in Escherichia coli and reduction of aerobic acetate formation. Appl Microbiol Biotechnol 2007, 74:406-421.

11. Varma A, Palsson BO: Stoichiometric flux balance models quantitatively predict growth and metabolic by-product secretion in wild-type Escherichia coli W3110. Appl Environ Microbiol 1994, 60:3724-3731.

12. Paalme T, Elken R, Kahru A, Vanatalu K, Vilu R: The growth rate control in Escherichia coli at near to maximum growth rates: the A-stat approach. Antonie van Leeuwenhoek 1997, 71:217-230.

13. Kayser A, Weber J, Hecht V, Rinas U: Metabolic flux analysis of Escherichia coli in glucose-limited continuous culture. I. Growth-rate-dependent metabolic efficiency at steady state. Microbiology 2005, 151:693-706.

14. El-Mansi M: Flux to acetate and lactate excretions in industrial fermentations: Physiological and biochemical implications. J Ind Microbiol Biotechnol 2004, 31:295-300.

15. De Mey M, De Maeseneire S, Soetaert W, Vandamme E: Minimizing acetate formation in E. coli fermentations. J Ind Microbiol Biotechnol 2007, 34:689-700.

16. El-Mansi EM, Holms WH: Control of carbon flux to acetate excretion during growth of Escherichia coli in batch and continuous cultures. J Gen Microbiol 1989, 135:2875-2883.

17. Yang Y-T, Bennett GN, San K-Y: Effect of inactivation of nuo and ackA-pta on redistribution of metabolic fluxes in Escherichia coli. Biotechnol Bioeng 1999, 65:291-297.

18. Dittrich CR, Vadali RV, Bennett GN, San K-Y: Redistribution of metabolic fluxes in the central aerobic metabolic pathway of $E$. coli mutant strains with deletion of the ackA-pta and poxB pathways for the synthesis of isoamyl acetate. Biotechnol Prog 2005, 21:627-631.

19. Abdel-Hamid AM, Attwood MM, Guest JR: Pyruvate oxidase contributes to the aerobic growth efficiency of Escherichia coli. Microbiology 2001, 147:1483-1498.

20. Phue J, Noronha SB, Hattacharyya R, Wolfe AJ, Shiloach J: Glucose metabolism at high density growth of $E$. coli $\mathrm{B}$ and $E$. coli $\mathrm{K}$ : differences in metabolic pathways are responsible for efficient glucose utilization in E. coli B as determined by microarrays and Northern blot analyses. Biotechnol Bioeng 2005, 90:805-820.

21. Castaño-Cerezo S, Pastor JM, Renilla S, Bernal V, Iborra JL, Cánovas M: An insight into the role of phosphotransacetylase (pta) and the acetate/ acetyl-CoA node in Escherichia coli. Microb Cell Fact 2009, 8:54.

22. Zhang W, Li F, Nie L: Integrating multiple 'omics' analysis for microbial biology: application and methodologies. Microbiology 2010, 156:287-301.

23. Paalme T, Kahru A, Elken R, Vanatalu K, Tiisma K, Vilu R: The computercontrolled continuous culture of Escherichia coli with smooth change of dilution rate. J Microbiol Methods 1995, 24:145-153.

24. Kasemets K, Drews M, Nisamedtinov I, Adamberg K, Paalme T: Modification of A-stat for the characterization of microorganisms. J Microbiol Methods 2003, 55:187-200.

25. Saier MH, Ramseier TO: The Catabolite Repressor/Activator (Cra) Protein of Enteric Bacteria. J Bacteriol 1996, 178:3411-3417.

26. Vemuri GN, Altman E, Sangurdekar DP, Khodursky AB, Eiteman MA: Overflow metabolism in Escherichia coli during steady-state growth: transcriptional regulation and effect of the redox ratio. Appl Environ Microbiol 2006, 72:3653-3661.

27. Ishii N, Nakahigashi K, Baba T, Robert M, Soga T, Kanai A, Hirasawa T, Naba M, Hirai K, Hoque A, Ho PY, Kakazu Y, Sugawara K, Igarashi S,
Harada S, Masuda T, Sugiyama N, Togashi T, Hasegawa M, Takai Y, Yugi K, Arakawa K, Iwata N, Toya Y, Nakayama Y, Nishioka T, Shimizu K, Mori H, Tomita M: Multiple high-throughput analyses monitor the response of $E$. coli to perturbations. Science 2007, 316:593-597.

28. Shin S, Chang D, Pan JG: Acetate Consumption Activity Directly Determines the Level of Acetate Accumulation During Escherichia coli W3110 Growth. J Microbiol Biotechnol 2009, 19:1127-1134.

29. Barak R, Abouhamad WN, Eisenbach M: Both acetate kinase and acetyl coenzyme A synthetase are involved in acetate-stimulated change in the direction of flagellar rotation in Escherichia coli. J Bacteriol 1998, 180:985-988.

30. Da Re SS, Deville-Bonne D, Tolstykh T, V ron M, Stock JB: Kinetics of CheY phosphorylation by small molecule phosphodonors. FEBS Lett 1999, 457:323-326.

31. Mayover TL, Halkides CJ, Stewart RC: Kinetic characterization of CheY phosphorylation reactions: comparison of P-CheA and small-molecule phosphodonors. Biochemistry 1999, 38:2259-2271.

32. Klein $A H$, Shulla A, Reimann Sa, Keating DH, Wolfe AJ: The intracellular concentration of acetyl phosphate in Escherichia coli is sufficient for direct phosphorylation of two-component response regulators. $J$ Bacteriol 2007, 189:5574-5581

33. Barak R, Welch M, Yanovsky A, Oosawa K, Eisenbach M: Acetyladenylate or its derivative acetylates the chemotaxis protein $\mathrm{CheY}$ in vitro and increases its activity at the flagellar switch. Biochemistry 1992, 31:10099-10107.

34. Yan J, Barak R, Liarzi O, Shainskaya A, Eisenbach M: In vivo acetylation of $\mathrm{CheY}$, a response regulator in chemotaxis of Escherichia coli. J Mol Biol 2008, 376:1260-1271.

35. Matsubara M, Mizuno T: EnvZ-independent phosphotransfer signaling pathway of the OmpR-mediated osmoregulatory expression of OmpC and OmpF in Escherichia coli. Biosci Biotechnol Biochem 1999, 63:408-414.

36. Anfora AT, Halladin DK, Haugen BJ, Welch RA: Uropathogenic Escherichia coli CFT073 is adapted to acetatogenic growth but does not require acetate during murine urinary tract infection. Infect Immun 2008, 76:5760-5767.

37. McCleary W, Stock J: Acetyl phosphate and the activation of 2component response regulators. J Biol Chem 1994, 269:31567-31572.

38. Feng J, Atkinson MR, McCleary W, Stock JB, Wanner BL, Ninfa AJ: Role of phosphorylated metabolic intermediates in the regulation of glutamine synthetase synthesis in Escherichia coli. J Bacteriol 1992, 174:6061-6070.

39. Mizrahi I, Biran D, Ron EZ: Involvement of the Pta-AckA pathway in protein folding and aggregation. Res Microbiol 2009, 160:80-84.

40. Wolfe AJ, Chang D-E, Walker JD, Seitz-Partridge JE, Vidaurri MD, Lange CF, Prüß BM, Henk MC, Larkin JC, Conway T: Evidence that acetyl phosphate functions as a global signal during biofilm development. Mol Microbiol 2003, 48:977-988.

41. Shi IY, Kuzminov A: A Defect in the Acetyl Coenzyme-Acetate Pathway Poisons Recombinational Repair-Deficient Mutants of Escherichia coli. J Bacteriol 2005, 187:1266-1275

42. Nyström T: The glucose-starvation stimulon of Escherichia coli: induced and repressed synthesis of enzymes of central metabolic pathways and role of acetyl phosphate in gene expression and starvation survival. Mol Microbiol 1994, 12:833-843.

43. Postma E, Verduyn C, Scheffers Wa, Van Dijken JP: Enzymic analysis of the crabtree effect in glucose-limited chemostat cultures of Saccharomyces cerevisiae. Appl Environ Microbiol 1989, 55:468-477.

44. Lin H, Castro NM, Bennett GN, San K: Acetyl-CoA synthetase overexpression in Escherichia coli demonstrates more efficient acetate assimilation and lower acetate accumulation: a potential tool in metabolic engineering. Appl Microbiol Biotechnol 2006, 71:870-874.

45. Rosenzweig F, Adams J: Microbial Evolution in a Simple Unstructured Environment: Genetic Differentiation in Escherichia coli. Genetics 1994 917:903-917.

46. Treves DS, Manning S, Adams J: Repeated evolution of an acetatecrossfeeding polymorphism in long-term populations of Escherichia coli. Mol Biol Evol 1998, 15:789-797.

47. Franchini AG, Egli T: Global gene expression in Escherichia coli K-12 during short-term and long-term adaptation to glucose-limited continuous culture conditions. Microbiology 2006, 152:2111-2127. 
48. Hardiman T, Lemuth K, Keller M, Reuss M, Siemannherzberg M: Topology of the global regulatory network of carbon limitation in Escherichia coli. J Biotechnol 2007, 132:359-374.

49. Görke B, Stülke JR: Carbon catabolite repression in bacteria: many ways to make the most out of nutrients. Nat Rev Microbiol 2008, 6:613-624.

50. Narang A: Quantitative effect and regulatory function of cyclic adenosine 5区-phosphate in Escherichia coli. J Biosci 2009, 34:445-463.

51. Nahku R, Valgepea K, Lahtvee PJ, Erm S, Abner K, Adamberg K, Vilu R: Specific growth rate dependent transcriptome profiling of Escherichia coli K12 MG1655 in accelerostat cultures. J Biotechnol 2010, 145:60-65.

52. Nanchen A, Schicker A, Revelles O, Sauer U: Cyclic AMP-dependent catabolite repression is the dominant control mechanism of metabolic fluxes under glucose limitation in Escherichia coli. J Bacteriol 2008, 190:2323-2330.

53. Khankal R, Chin JW, Ghosh D, Cirino PC: Transcriptional effects of CRP* expression in Escherichia coli. J Biol Eng 2009, 3:13.

54. Ishizuka H, Hanamura A, Inada T, Aiba H: Mechanism of the downregulation of cAMP receptor protein by glucose in Escherichia coli: role of autoregulation of the crp gene. EMBO J 1994, 13:3077-3082.

55. Sarkar D, Siddiquee KA, Araúzo-Bravo MJ, Oba T, Shimizu K: Effect of cra gene knockout together with edd and iclR genes knockout on the metabolism in Escherichia coli. Arch Microbiol 2008, 190:559-751.

56. Adamberg K, Lahtvee PJ, Valgepea K, Abner K, Vilu R: Quasi steady state growth of Lactococcus lactis in glucose-limited acceleration stat (A-stat) cultures. Antonie van Leeuwenhoek 2009, 95:219-226.

57. Martens L, Hermjakob H, Jones P, Adamski M, Taylor C, States D, Gevaert K, Vandekerckhove J, Apweiler R: PRIDE: the proteomics identifications database. Proteomics 2005, 5:3537-3545.

58. Barsnes H, Vizcaíno JA, Eidhammer I, Martens L: PRIDE Converter: making proteomics data-sharing easy. Nat Biotechnol 2009, 27:598-599.

doi:10.1186/1752-0509-4-166

Cite this article as: Valgepea et al:: Systems biology approach reveals that overflow metabolism of acetate in Escherichia coli is triggered by carbon catabolite repression of acetyl-CoA synthetase. BMC Systems Biology 2010 4:166

\section{Submit your next manuscript to BioMed Central and take full advantage of:}

- Convenient online submission

- Thorough peer review

- No space constraints or color figure charges

- Immediate publication on acceptance

- Inclusion in PubMed, CAS, Scopus and Google Scholar

- Research which is freely available for redistribution

Submit your manuscript at www.biomedcentral com/submit 